\title{
ANÁLISE DE RESÍDUOS DO INSETICIDA METAMIDOFÓS EM SOJA E DETERMINAÇÃO FINAL POR CROMATOGRAFIA EM FASE GASOSA
}

\author{
FRANCISCO JOSÉ C. LIMA* \\ SÔNIA MARIA CARVALHO NEIVA TANAKA** \\ GILVANDA SILVA NUNES** \\ TERESA CRISTINA RODRIGUES DOS SANTOS** \\ PAULO JORGE MARQUES CORDEIRO***
}

\begin{abstract}
Neste trabalho propõe-se método rápido de procedimentos de extração e clean up para determinações do inseticida Metamidofós em amostras de soja. Análises de amostras de soja seca e hidratada, fortificadas em níveis de $5 \mu \mathrm{g} \mathrm{g}{ }^{-1}$, apresentaram recuperação média de $93-94 \%$ e coeficiente de variação de 17 a $31 \%$ com os sistemas adsorvente alumina/florisil e eluente metanol/ acetonitrila 1:3. Testes empregando sílica 15\% desativada também mostraram eficiência com recuperação final de, aproximadamente, $75 \%$ e coeficientes de variação entre 18 a $27 \%$. Cromatogramas com mínima interferência, nenhum efeito de matriz e boa eficiência na extração foram obtidos com a aplicação do método proposto.
\end{abstract}

PALAVRAS-CHAVE: INSETICIDA METAMIDOFÓS; SOJA; CROMATOGRAFIA EM FASE GASOSA; CROMATOGRAFIA POR ADSORÇÃO.

* $\quad$ Mestre em Química pela Universidade Federal do Maranhão (UFMA); Trabalho desenvolvido no Núcleo de Análises de Resíduos de Pesticidas (NARP), CCET/UFMA.

** Professores, Doutores, Departamento de Tecnologia Química, UFMA, Bacanga, São Luís - MA (e-mail:sonia@ufma.br).

*** Pesquisador, Instituto de Química de São Carlos, Universidade de São Paulo, São Carlos - SP. 


\section{INTRODUÇÃO}

A utilização de compostos sulfurados foi um dos primeiros recursos utilizados pelo homem para garantir boas colheitas. Posteriormente, foram lançados no mercado compostos orgânicos sintéticos com propriedades letais para vários tipos de pestes que atacam as colheitas, sendo o diclorodifeniltricloroetano (DDT), um dos mais eficientes. Diferentes pesticidas, com propriedades específicas e ações eficazes, foram desenvolvidos, encontrando-se os compostos organofosforados entre os agrotóxicos mais utilizados para controle de insetos $(1,2)$. Por outro lado, a utilização de grandes quantidades desses agroquímicos em diversas culturas, principalmente nas que ocupam grandes extensões, como a de soja, tem sido motivo de preocupação devido aos impactos nocivos sobre o meio ambiente e aos seres humanos.

O Metamidofós é um pesticida (inseticida e acaricida) organofosforado muito utilizado no controle de insetos e pestes para a proteção de culturas (3-6), mas que apresenta vários riscos à saúde humana e aos animais devido à sua elevada toxicidade (4-7). Muitos estudos têm sido realizados no sentido de verificar e quantificar os efeitos e o mecanismo de ação do Metamidofós no meio ambiente (8-11), em matrizes vegetais $(2,4,11-15)$ e ao homem $(5,16-18)$.

A maioria dos métodos de análises de pesticidas envolvendo a detecção cromatográfica emprega (como uma das etapas de bancada) a cromatografia de adsorção para purificação de extratos antes da determinação cromatográfica propriamente dita. O uso de cartuchos de extração por fase sólida (SPE) é mais comum, apesar de dispendioso para grande parte dos laboratórios. O preparo de colunas de adsorção a partir do empacotamento (preenchimento) com fase adsorvente adequada à polaridade do(s) composto(s) constitui alternativa aos procedimentos de SPE. Sua eficiência foi comprovada em estudos anteriores na purificação de extratos contendo compostos de polaridade média à alta $(3,6)$.

A cromatografia em fase gasosa é muito utilizada nas análises de resíduos de Metamidofós, seja variando o detector ou os instrumentos de injeção ou, ainda, acoplando outro instrumento para aumentar sua sensibilidade. Essa técnica proporciona resultados satisfatórios em análises de pesticidas em diferentes matrizes (1-2,19-27).

O presente trabalho apresenta metodologia rápida para extração do inseticida Metamidofós e purificação simultânea dos extratos de soja, utilizando a cromatografia em fase gasosa.

\section{MATERIAL E MÉTODOS}

\subsection{SOLVENTES, ÁGUA E SOLUÇÃO DE TRABALHO}

Os solventes utilizados foram de grau resíduo ou HPLC (Merck, pureza > 98\%). Em todos os procedimentos utilizou-se água destilada e purificada em sistema Milli-Q Academic da Millipore.

As soluções de trabalho do inseticida Metamidofós com concentração $5,0 \mu \mathrm{g} \mathrm{mL}^{-1}$ foram preparadas a partir da diluição, com metanol, de solução estoque com padrão certificado a $154,38 \mu \mathrm{g} \mathrm{mL} \mathrm{m}^{-1}$ de metanol com 99\% de pureza (Dr. Ehrnstorfer, Alemanha).

\subsection{LIMPEZA DA VIDRARIA}

A vidraria utilizada foi lavada, seqüencialmente, com solução Extran Alcalino ${ }^{\circledR}$ a $20 \%$ (Merck), água em abundância e, por fim, com acetona p.a.

As colunas de vidro utilizadas na extração/purificação foram imersas em Extran alcalino por $48 \mathrm{~h}$ e lavadas com água. Esse procedimento foi necessário para evitar contaminações que pudessem influenciar nos resultados das análises. 


\subsection{FORTIFICAÇÃO DA AMOSTRA DE SOJA}

Amostras com teor natural de umidade e amostras previamente secas em estufa a $105^{\circ} \mathrm{C}$ foram fortificadas antes da preparação da coluna. Durante a homogeneização foram misturados a amostra e o pesticida que permaneceram em repouso por 10 minutos. Após pesagem dos adsorventes, esses foram incorporados à mistura sendo, em seguida, preparada a coluna de adsorção. A concentração empregada nos ensaios de fortificação foi de $1,0 \mu \mathrm{g} \mathrm{mL}^{-1}$, a partir da adição da solução de trabalho em massa apropriada da soja finamente pulverizada em moinho de bolas.

\subsection{PREPARO DOS ADSORVENTES}

Os adsorventes alumina, sílica e florisil (atividade I Korngro, 70-230 mesh ASTM, Merck) foram secos a $105^{\circ} \mathrm{C}$ por $1 \mathrm{~h}$ e resfriados em dessecador. Em seguida, cada adsorvente foi parcialmente desativado por adição de água em diferentes proporções percentuais em relação à sua massa: $3 \% \mathrm{e}$ $6 \%$ para a alumina e $15 \%$ para a sílica. O adsorvente florisil não sofreu desativação. Após homogeneização e repouso por $24 \mathrm{~h}$, o material (desativado ou não) foi utilizado no tempo máximo de 5 dias, tendo sido armazenado ao abrigo da luz. Maiores detalhes dos procedimentos utilizados no preparo dos adsorventes estão descritos no trabalho de NUNES (12).

\subsection{TESTES DE RECUPERAÇÃO DO METAMIDOFÓS}

A alumina $3 \%$ ativada foi secada em estufa a $105^{\circ} \mathrm{C}$ por $1 \mathrm{~h}$ e resfriada em dessecador. Em seguida, o adsorvente foi parcialmente desativado por adição de água na proporção de $3 \%$ em relação à sua massa. Após homogeneização e repouso, por $24 \mathrm{~h}$, ao abrigo da luz, o material foi utilizado. $\mathrm{O}$ mesmo procedimento foi utilizado para obtenção da alumina $6 \%$ desativada. Para florisil procedeu-se apenas à secagem em estufa a $105^{\circ} \mathrm{C}$ por $1 \mathrm{~h}$. A sílica gel 60 foi inicialmente ativada em estufa a $130^{\circ} \mathrm{C}$ por $24 \mathrm{~h}$ e depois resfriada em dessecador. $\mathrm{O}$ adsorvente foi parcialmente desativado por adição de água na proporção de $15 \%$ em relação à sua massa. Após homogeneização e repouso por $24 \mathrm{~h}$, ao abrigo da luz, o material foi utilizado. A recuperação foi feita em duas etapas, utilizando-se os adsorventes SA-1 (alumina a 3\% desativada/florisil 3:1), SA-2 (alumina a 6\% desativada) e SA-3 (sílica a $15 \%$ desativada). Na primeira etapa foram efetuados testes com os três sistemas visando verificar qual dos adsorventes apresentaria melhor índice de recuperação para misturá-lo à amostra na segunda etapa. O processo de preparação, tanto da coluna quanto dos adsorventes, foi o mesmo na primeira e segunda etapas. A presença da amostra real ocorreu apenas na segunda etapa (12).

\subsection{PREPARO DA AMOSTRA}

A soja utilizada no presente trabalho foi fornecida pela EMBRAPA/MA, cultivada sem a utilização de pesticidas ou qualquer outro tipo de produto usualmente empregado no controle de insetos, doenças ou ervas daninhas.

Experimentos foram realizados com amostras de soja contendo teor natural de umidade ("soja úmida") e também com "soja desidratada" em ultracentrífuga a vácuo da Lab Com (EUA), com redução de umidade de cerca de $10 \%$ em peso.

$\mathrm{Na}$ fortificação da amostra com o pesticida, a soja foi previamente triturada e mantida sob refrigeração para conservação das propriedades naturais até o momento de sua utilização. Pouco antes de ser misturada com o adsorvente foi adicionado o pesticida Metamidofós à amostra na concentração final de $1 \mu \mathrm{g} \mathrm{g}{ }^{-1}$ de soja. A soja fortificada permaneceu em repouso por 15 minutos antes do preparo da coluna adsorvente. 


\subsection{PREPARO DAS COLUNAS DE ADSORÇÃO HOME-GLASSE PROCEDIMENTOS DE ELUIÇÃO}

Os sistemas eluentes (SE) foram preparados a partir da mistura de metanol e acetonitrila nas proporções 1:3, 1:5 e 1:1 (v:v) designadas como SE-1, SE-2 e SE-3, respectivamente.

Para a eluição foram utilizadas colunas de vidro Pyrex® de $200 \mathrm{~mm}$ de comprimento e $25 \mathrm{~mm}$ de diâmetro interno. O "empacotamento" de cada coluna ocorreu por adição cuidadosa da mistura adsorvente/amostra, ambas na mesma proporção. Porção de $2 \mathrm{~g}$ da mistura de adsorvente com a soja triturada foi usada para o enchimento da coluna de vidro. As colunas foram acondicionadas com $15 \mathrm{~mL}$ do sistema eluente, adicionados em duas etapas (inicialmente $10 \mathrm{~mL}$ e, após $5 \mathrm{~min}$, mais $5 \mathrm{~mL}$ ). $\mathrm{O}$ procedimento de eluição consistiu na adição de $1 \mathrm{~mL}$ da solução-padrão de Metamidofós, com concentração de $5 \mu \mathrm{g} \mathrm{g}^{-1}$ para testes com os adsorventes e de $1 \mu \mathrm{g} \mathrm{g}^{-1}$ para os testes com a amostra. O eluato (aproximadamente, $15 \mathrm{~mL}$ ) foi recolhido e concentrado em evaporador rotativo (QUIMIS, modelo 218.2), em temperatura entre 35 e $45^{\circ} \mathrm{C}$. Temperaturas maiores não foram utilizadas para evitar a degradação do composto, embora esse não seja termolábil (2). O resíduo foi retomado com $1 \mathrm{~mL}$ de acetonitrila (testes com adsorventes) e acetato de etila (testes com a mistura adsorvente/ soja). Após seleção das melhores combinações dos sistemas eluentes e sistemas adsorventes, 8 eluições foram realizadas com os sistemas que apresentam melhor eficiência. Ao final de cada procedimento de extração/purificação on line, os extratos finais foram filtrados em filtro de poliestireno com $0,45 \mu \mathrm{m}$ de diâmetro de poro (Millex, Millipore), antes de serem injetados no cromatógrafo a gás.

\subsection{ANÁLISES CROMATOGRÁFICAS}

Os cromatogramas foram obtidos em cromatógrafo a gás (GC) SHIMADZU, modelo GC-17 A, com detector fotométrico de chama (FPD). As condições cromatográficas foram: a) gás de arraste nitrogênio; b) fluxo do gás de arraste $-1 \mathrm{~mL} \mathrm{~min}^{-1}$, com velocidade linear de $20 \mathrm{~cm} \mathrm{~s}^{-1}$; c) coluna - HP1 metilsilicone ( $25 \mathrm{~m}$ de comprimento e $0,22 \mathrm{~mm}$ de diâmetro interno); d) espessura do filme $0,33 \mu \mathrm{m} ; \mathrm{e}$ ) temperatura do detector $-300^{\circ} \mathrm{C}$; f) temperatura do injetor $-300^{\circ} \mathrm{C} ; \mathrm{g}$ ) razão Split - $1: 30 ; \mathrm{h}$ ) temperatura inicial: $100^{\circ} \mathrm{C}$; i) rampa de temperatura $-10^{\circ} \mathrm{C} \mathrm{min}^{-1}$; j) tempo final $-5 \mathrm{~min}$, e k) pressão na cabeça da coluna - $140 \mathrm{kPa}$.

Para cada amostra de extrato analisada foram realizadas duas injeções, sendo tomadas (para efeito de cálculos de concentração) as médias das áreas obtidas. A curva analítica foi elaborada a partir de soluções com concentrações no intervalo 0-10 $\mathrm{g} \mathrm{mL}^{-1}$, preparadas a partir de diluições da solução estoque de Metamidofós com metanol.

\subsection{ANÁLISES ESTATÍSTICAS}

As análises estatísticas foram efetuadas pela comparação de médias dos dados de recuperação, obtidos a partir das eluições efetuadas com as melhores combinações de sistemas adsorventes e eluentes, utilizando-se o adsorvente misturado à soja úmida e desidratada (8 repetições para cada sistema). Para a comparação de médias foi empregado o Teste $F(28)$.

\section{RESULTADOS E DISCUSSÃO}

\subsection{SENSIBILIDADE E LINEARIDADE}

O limite de detecção (relativo ao instrumento) encontrado foi de $9 \mu \mathrm{g} \mathrm{L}^{-1}$. A linearidade ficou entre 10 e $5.000 \mu \mathrm{g} \mathrm{L}^{-1}$. Tal faixa de concentração mostrou-se adequada para análises do composto em níveis de concentração, relativamente elevados até teores-traço. 


\subsection{RECUPERAÇÕES DO COMPOSTO METAMIDOFÓS EM AMOSTRAS- PADRÃO, APÓS ELUIÇÕES EM COLUNAS HOME-GLASS}

A Figura 1 ilustra o cromatograma do Metamidofós, após a eluição com o sistema eluente SE1, em coluna preparada com o sistema adsorvente SA-1. O tempo de retenção correspondente ao pico do Metamidofós pode ser visto nitidamente em torno de $7 \mathrm{~min}$. Não houve sobreposição de picos e os co-extrativos, provavelmente associados com o adsorvente, não comprometeram a identificação do pico desejado. A recuperação média para análise em duplicata foi de 101,2\%. O sistema SA-1, combinado com o SE-2, também apresentou boa recuperação, com média de aproximadamente $93,7 \%$ e coeficiente de variação (CV) de 12,6\% (Tabela 1). Confirmou-se assim o bom desempenho do sistema

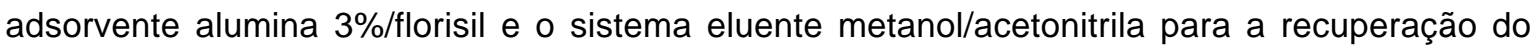
Metamidofós. Todavia, quando o sistema eluente empregado foi o SE-3, contendo menor proporção de acetonitrila, observou-se diminuição para $80,8 \%$ na recuperação, bem como aumento no valor do coeficiente de variação (CV) para 22\% (Tabela 1).

FIGURA 1 - CROMATOGRAMA DO METAMIDOFÓS, APÓS ELUIÇÃO EM COLUNA DE SA-1 (ALUMINA 3\% DESATIVADA/FLORISIL 3:1). COLUNA CROMATOGRÁFICA: HP-1 METILSILICONE; COMPRIMENTO: $25 \mathrm{~m}$; DIÂMETRO INTERNO: 0,22 mm; ESPESSURA DO FILME: 0,33 $\mu \mathrm{m}$; GÁS DE ARRASTE: NITROGÊNIO; TEMPERATURA DA COLUNA: $300^{\circ} \mathrm{C}$; TEMPERATURA DO DETECTOR: $300^{\circ} \mathrm{C}$

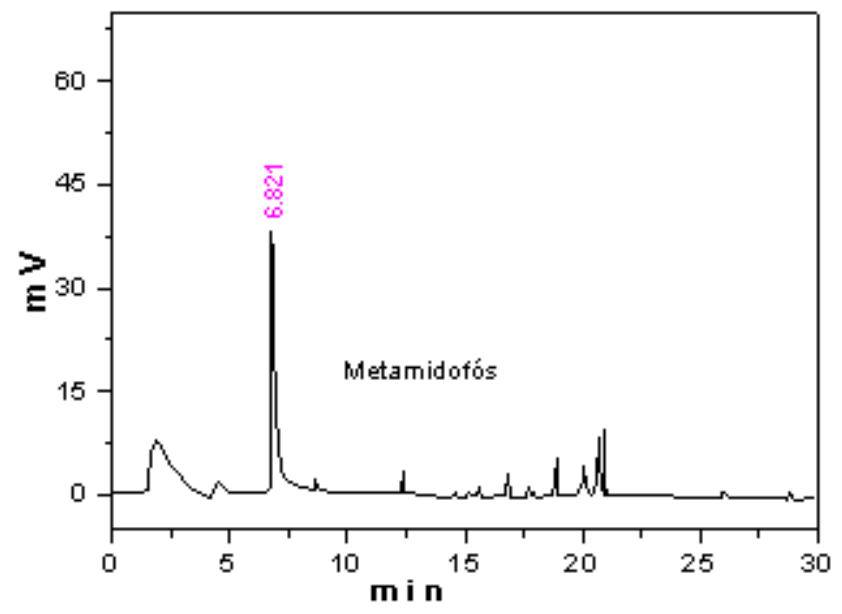

TABELA 1 - RECUPERAÇÕES DO COMPOSTO METAMIDOFÓS EM COLUNA CONTENDO O SISTEMA ADSORVENTE SA-1 (ALUMINA 3\%/FLORISIL 3:1) E DIFERENTES SISTEMAS ELUENTES

\begin{tabular}{lcc}
\hline Sistema Eluente (SE) & $\begin{array}{c}\text { Mødia de } \\
\text { Recupera a o (\%) }\end{array}$ & $\begin{array}{c}\text { Coeficiente de } \\
\text { Varia a o (\%) }\end{array}$ \\
\hline metanol/acetonitrila 1:3 (SE-1) & 101,2 & 15,2 \\
metanol/acetonitrila 1:5 (SE-2) & 93,7 & 12,6 \\
metanol/acetonitrila 1:1 (SE-3) & 80,8 & 22,0 \\
\hline
\end{tabular}

${ }^{*}$ Com base em duas repetições. 
O bom comportamento do SA-1 com SE-1 e SE-2 pode ser atribuído à interação da polaridade do adsorvente com os sistemas eluentes. A diferença de polaridade não é muito grande entre os dois sistemas eluentes, porém as proporções, em comparação com a combinação 1:1 do SE-3, são grandes (provavelmente, devido à diferença acentuada de polaridade em relação ao pesticida que foi arrastado na eluição).

Em relação à moderada polaridade do Metamidofós, o SE-2 resultou em boa capacidade de eluição podendo ser empregado para outros pesticidas de elevada polaridade (tais como carbamatos e piretróides). O SA-1 apresentou bom comportamento, quando associado a todos os sistemas eluentes. Sua recuperação média variou entre $80,8 \%$ e 101,2\%, estando em concordância com valores reportados na literatura para o mesmo pesticida (29-31). Esses valores de recuperação indicam que a interação das polaridades do adsorvente com as moléculas do pesticida propiciaram conjunto pesticidaadsorvente favorável à interação com o SE-1. Assim, foi possível promover completo arraste do pesticida que se encontrava aderido à superfície do adsorvente (24). Por outro lado, a queda na recuperação quando a quantidade de acetonitrila na mistura foi diminuída de 1:3 (SE-1) ou 1:5 (SE-2) para 1:1 (SE3) evidenciou maior capacidade de interação desse solvente com o adsorvente na etapa de eluição do composto (32).

$\mathrm{Na}$ Tabela 2 são apresentados os valores médios de recuperação e coeficientes de variação para as extrações do Metamidofós em colunas contendo o sistema adsorvente SA-2 (com os mesmos sistemas eluentes anteriores). A eluição com o sistema SE-1 evidenciou a menor recuperação (cerca de $85,8 \%$ ), provavelmente em razão da Alumina $6 \%$ desativada apresentar como característica principal a presença de grupos $\mathrm{OH}$ desativados na superfície do adsorvente. Tal fato facilitou a eluição e dificultou a sua hidrólise, acarretando perdas na recuperação $(32,33)$. A diminuição na relação metanol/acetonitrila de 1:3 para 1:1 apresentou recuperação média de $100 \%$, porém com CV relativamente alto (42\%) pela diferença de resultados entre as duas determinações, evidenciando perda na eficiência do processo de extração do pesticida. Por outro lado, o aumento na proporção de acetonitrila para 1:5 permitiu não somente manter boa média de recuperação (108\%), mas também reduzir o valor do CV para $16 \%$.

\section{TABELA 2 - RECUPERAÇÕES DO COMPOSTO METAMIDOFÓS EM COLUNA CONTENDO O SISTEMA ADSORVENTE SA-2 (ALUMINA 6\%) E DIFERENTES SISTEMAS ELUENTES}

\begin{tabular}{lcc}
\hline Sistema Eluente (SE) & $\begin{array}{c}\text { Mdia de } \\
\text { Recupera } \\
(\%)\end{array}$ & $\begin{array}{c}\text { Coeficiente de } \\
\text { Varia } \mathbf{0}(\%)^{*}\end{array}$ \\
\hline Metanol/acetonitrila $1: 3(\mathrm{SE}-1)$ & 85,8 & 20,0 \\
Metanol/acetonitrila $1: 5(\mathrm{SE}-2)$ & 107,6 & 16,0 \\
Metanol/acetonitrila $1: 1(\mathrm{SE}-3)$ & 100,0 & 42,0 \\
\hline
\end{tabular}

*Com base em duas repetições.

A Tabela 3 mostra que o terceiro sistema adsorvente investigado, SA-3 (sílica 15\% desativada), apresentou boa eficiência quando associado com os sistemas eluentes SE-1 (recuperação média de 99,5\%) e o SE-3 (recuperação média de 108,5\%). Muito embora em associação com o SE-2, a recuperação média tenha sido de $122,4 \%$ com CV de $6,2 \%$, esse resultado pode também ser considerado satisfatório em análise de resíduos. 


\begin{tabular}{lcc}
\hline Sistema Eluente & $\begin{array}{c}\text { Mødia de } \\
\text { Recupera } \text { a o (\%) }\end{array}$ & $\begin{array}{c}\text { Coeficiente de } \\
\text { Varia a o (\%) }\end{array}$ \\
\hline metanol/acetonitrila 1:3 (SE-1) & 99,5 & 20,0 \\
metanol/acetonitrila 1:5 (SE-2) & 122,4 & 6,2 \\
metanol/acetonitrila 1:1 (SE-3) & 108,5 & 11,0 \\
\hline
\end{tabular}

${ }^{*}$ Com base em 2 repetições.

\subsection{RECUPERAÇÕES DO COMPOSTO METAMIDOFÓS EM AMOSTRAS DE SOJA APÓS ELUIÇÕES EM COLUNAS HOME-GLASS}

A eficiência prática das colunas de adsorção para recuperação do composto Metamidofós foi testada em amostras de soja úmida e soja desidratada. Foram utilizados os sistemas adsorventes SA-1 e SA-3 por terem apresentado bons resultados de recuperação e coeficientes de variação relativamente mais baixos, bem como o sistema eluente SE-1 devido a melhor interação com os dois adsorventes.

A adequada eficiência do sistema adsorvente SA-1, combinado com o sistema eluente SE-1 para amostras de soja contendo teor natural de umidade, foi caracterizada pela recuperação média de $93 \%$ e pequenas variações nas recuperações individuais (Tabela 4). Essas, provavelmente devem-se às diferentes interações da amostra com o adsorvente e ao fluxo de eluição que (apesar do rígido controle para se manter em torno de $2 \mathrm{~mL} \mathrm{~min}^{-1}$ ), apresentou variação de coluna para coluna. Tal efeito já foi relatado em estudos similares $(12,13)$.

\section{TABELA 4 - RECUPERAÇÕES DO COMPOSTO METAMIDOFÓS PARA AMOSTRAS DE SOJA ÚMIDA E DESIDRATADA EM COLUNA CONTENDO O SISTEMA ADSORVENTE SA-1 E SISTEMA ELUENTE SE-1}

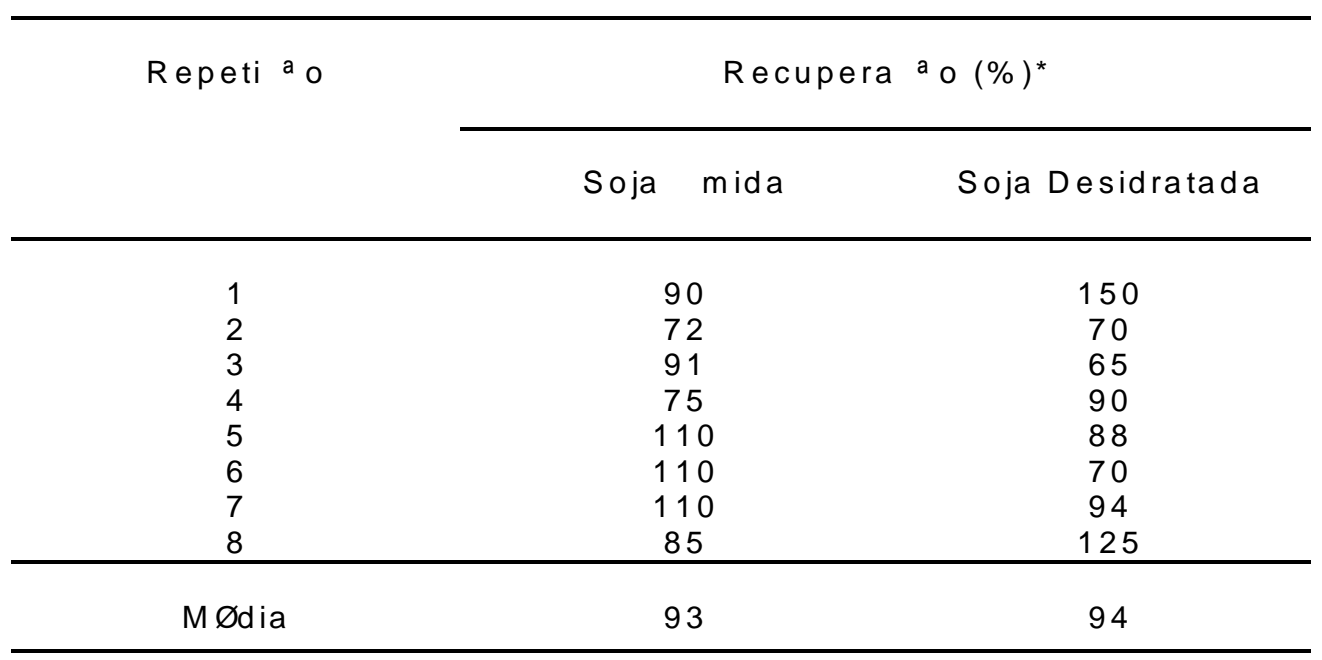

${ }^{*}$ Coeficiente de Variação $=16,8 \%$ para soja úmida e $31 \%$ para soja desidratada; $(n=8)$. 
A recuperação média obtida com a soja desidratada utilizando-se os sistemas SA-1 e SE-1 também foi bastante satisfatória (valor médio de $94 \%$ para 8 repetições), porém com CV elevado (31\%) (Tabela 4). A Figura 2 apresenta os cromatogramas obtidos com o SE-1 e o SA-3 misturados às amostras de soja úmida (a) e desidratada (b). Os cromatogramas apresentaram-se livres de interferências no tempo de retenção do pesticida. A comparação dos dados obtidos com soja úmida e soja desidratada indicou que o procedimento de extração da umidade não afetou a recuperação final do analito. Entretanto, o processo é lento e pode ter provocado diminuição na precisão da análise (CV maior, no caso da soja seca), já que a retirada de umidade não foi uniforme. Ficou evidente a ausência de outros picos que poderiam afetar a identificação do Metamidofós, durante a eluição do composto ao longo da coluna. De fato, estudo anterior (3) mostrou que a quantidade de água na amostra vegetal resultou na formação de emulsões. Para grãos em que o teor de umidade é relativamente baixo não ocorrem alterações nos resultados de recuperação, uma vez que a água presente é insuficiente para provocar emulsões no material adsorvente e reduzir a recuperação do composto em estudo (12).
FIGURA 2 - CROMATOGRAMAS DO METAMIDOFÓS MISTURADO À SOJA ÚMIDA (A) E DESIDRATADA (B), APÓS ELUIÇÃO EM COLUNA DE SA-3 (SÍLICA 15\% DESATIVADA). COLUNA CROMATOGRÁFICA: HP-1 METILSILICONE; COMPRIMENTO: 25 m; DIÂMETRO INTERNO: 0,22 mm; ESPESSURA DO FILME: 0,33 $\mu \mathrm{m}$; GÁS DE ARRASTE: NITROGÊNIO; TEMPERATURA DA COLUNA: $300^{\circ} \mathrm{C}$; TEMPERATURA DO DETECTOR: $300^{\circ} \mathrm{C}$
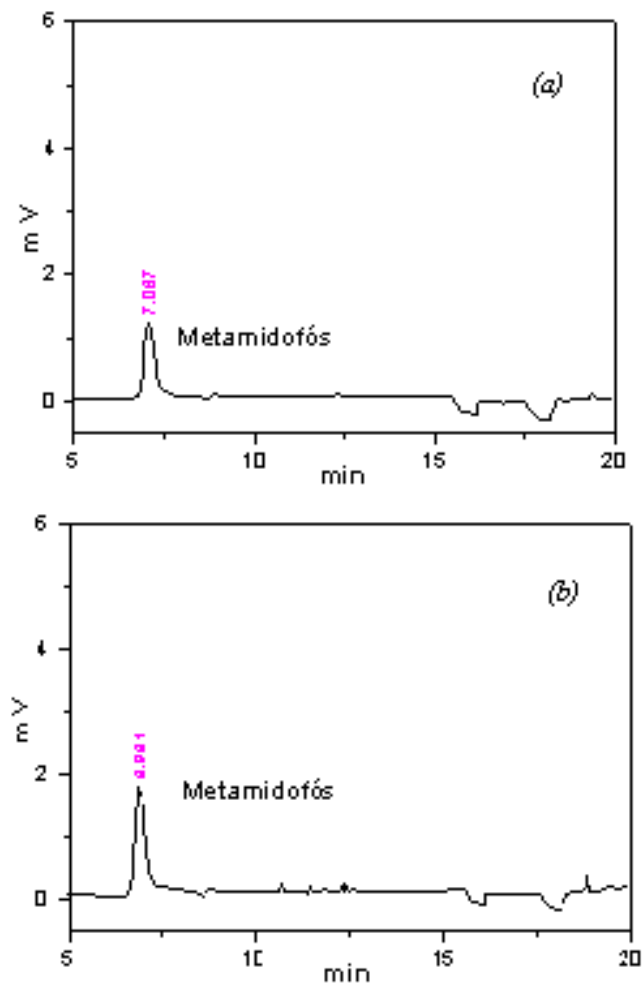

A combinação dos sistemas SA-3 e SE-1 apresentou flutuações consideráveis nos valores de recuperação e valor médio de $\sim 75 \%$, tanto para as amostras de soja úmida quanto desidratadas (Tabela 5). Portanto, a combinação não é apropriada para análises de contaminantes em níveis de traço (Tabela 5). Essa elevada variação nos valores de recuperação individuais deve-se à emulsificação do sistema adsorvente provocada pela presença de água, uma vez que a sílica apresenta maior capacidade para reter água que a alumina. As amostras desidratadas, além da recuperação média de 
$\sim 76 \%$ apresentaram alto valor de CV (27\%). Tal fato revelou desvantagem do processo de secagem da soja, que além de demorado (etapa adicional no processo de extração e purificação), pode conduzir a perdas de composto não-estável em elevadas temperaturas.

TABELA 5 - RECUPERAÇÕES DO COMPOSTO METAMIDOFÓS PARA AMOSTRAS DE SOJA ÚMIDA E DESIDRATADA EM COLUNA CONTENDO O SISTEMA ADSORVENTE SA-3 E SISTEMA ELUENTE SE-1

\begin{tabular}{ccc}
\hline & \multicolumn{2}{c}{ Recupera ${ }^{2}$ o $(\%)^{*}$} \\
\cline { 2 - 3 } & Soja mida & Soja Desidratada \\
& & \\
\hline $\mathbf{1}$ & 93 & $--^{\mathrm{a}}$ \\
$\mathbf{3}$ & 84 & 60 \\
$\mathbf{4}$ & 82 & 94 \\
$\mathbf{5}$ & 81 & 62 \\
$\mathbf{6}$ & 57 & 75 \\
$\mathbf{7}$ & 81 & 70 \\
$\mathbf{8}$ & 65 & 70 \\
Mødia & 56 & 98 \\
\hline
\end{tabular}

${ }^{*}$ Coeficiente de Variação $=18,2 \%$ para soja com teor natural de umidade $(n=8)$ e $27 \%$ para soja desidratada $(n=7)$. a Valor discrepante no dado de recuperação $n^{\circ} 1$ para soja desidratada justificou a sua remoção no cálculo da recuperação média e do CV.

\subsection{COMPARAÇÃO ESTATÍSTICA DOS MÉTODOS TESTADOS}

As médias dos resultados obtidos para o tratamento $A$, procedimento de extração e purificação com SA-1, não diferiam entre si, sendo os procedimentos semelhantes (Tabela 6).

TABELA 6 - COMPARAÇÃO DAS MÉDIAS DE RECUPERAÇÃO PARA OS DIFERENTES MÉTODOS PROPOSTOS PARA PROCEDIMENTOS DE EXTRAÇÃO E PURIFICAÇÃO SIMULTÂNEAS (MÉTODOS ON LINE)

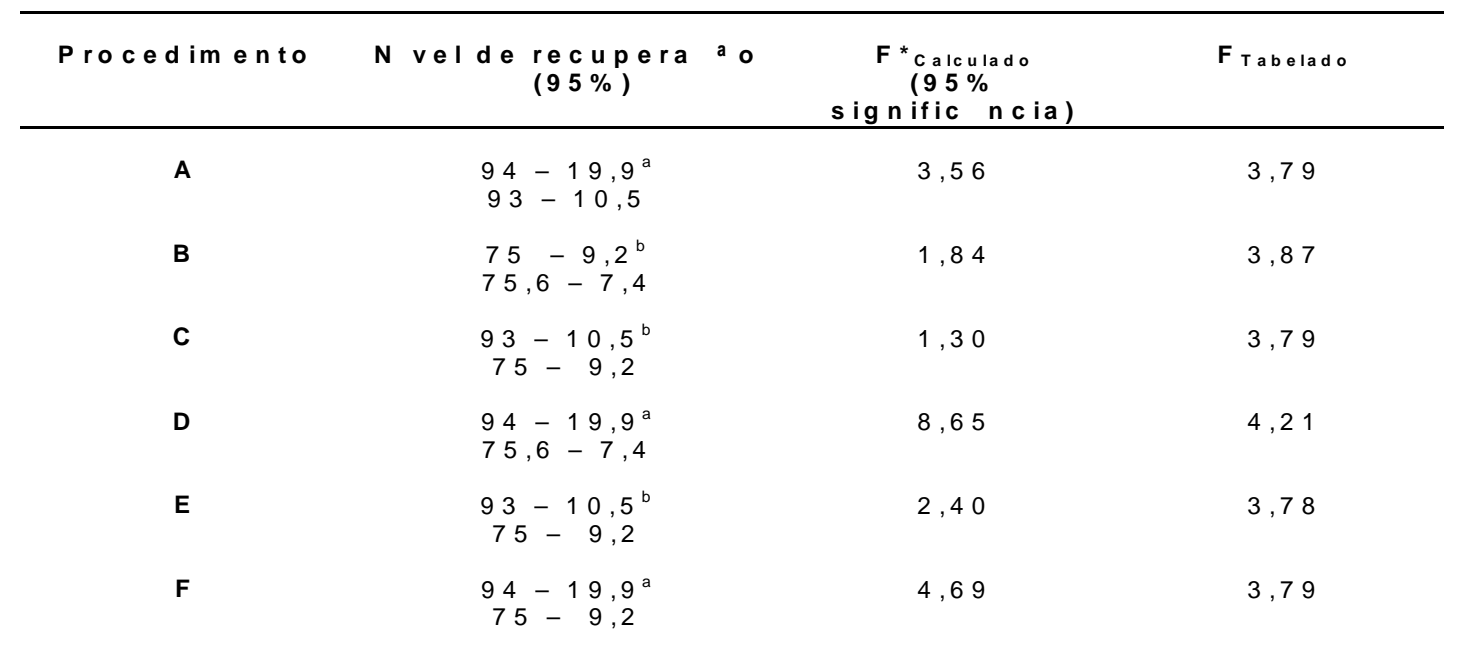

Procedimentos: $A$ = SA-1(úmida)xSA-1(desidratada); B = SA-3(úmida)xSA-3(desidratada); C = SA-1 (úmida)xSA3(úmida); $\mathrm{D}=\mathrm{SA}-1$ (desidratada)xSA3(Desidratada); $\mathrm{E}=\mathrm{SA}-1$ (úmida)xSA-3(desidratada); $\mathrm{F}=\mathrm{SA}-1$ (desidratada)xSA-3(úmida).

* Médias seguidas pela mesma letra não diferem estatisticamente no nível de significância de 95\%, segundo o teste F. 
A extração da umidade da soja, quando empregados o SA-1 e o SE-1, não influenciou diretamente os resultados de recuperação do Metamidofós. Da mesma forma, os procedimentos $\mathrm{D}$ e $\mathrm{F}$ apresentaram semelhança em suas médias. $O$ teste $F$ refere-se à comparação de procedimentos com diferentes adsorventes e teor natural de umidade na soja, contudo, os resultados encontrados mostraram-se estatisticamente semelhantes. Os procedimentos B, C e E com SA-3 e a soja com e sem umidade, apresentaram diferença na comparação de suas médias. Portanto, os resultados alcançados no processo de extração e purificação são diferentes (tratamento B), apesar das médias serem semelhantes e ter sido empregado o mesmo sistema adsorvente. A extração da umidade, neste caso, diminui a eficiência do processo.

\title{
4 CONCLUSÃO
}

Os três sistemas adsorventes testados, somente com o padrão do pesticida, apresentaram boa eficiência no processo de extração e purificação do Metamidofós. O sistema SA-1 apresentou melhores resultados de recuperação, tanto para a amostra de soja com teor natural de umidade como para a amostra de soja desidratada. Também revelou bons resultados, quando a eluição foi efetuada com o sistema eluente SE-1, (melhor eficiência na extração e purificação do Metamidofós) com reflexos, sobretudo, na elevada média da recuperação e adequação aos diferentes sistemas adsorventes. A extração de umidade da soja ocasionou redução no percentual de recuperação do pesticida. Os procedimentos de extração e purificação do Metamidofós com o SA-1 foram satisfatórios, considerando os resultados de recuperação e coeficientes de variação.

O consumo de reagentes foi pequeno e o tempo máximo para a obtenção dos extratos purificados foi de 35 minutos. O emprego das colunas home glass nesse método on line de extração/purificação mostrou-se satisfatório, permitindo a análise de dezenas de amostras no mesmo dia. Todavia, em comparação com os procedimentos de extração em fase sólida (SPE) que empregam cartuchos (e conseqüentemente, não necessitam de preparo de colunas) o método proposto não é adequado para análises de rotina com elevado número de amostras. Além disso, os cromatogramas obtidos em todos os testes apresentaram pouca interferência por coextrativos, baixo nível de ruído, nenhum efeito de matriz sobre a linha de base e boa reprodutibilidade dos tempos de retenção.

\section{ABSTRACT}

\begin{abstract}
METHAMIDOPHOS INSECTICIDE RESIDUE ANALYSIS IN SOYBEAN AND FINAL DETERMINATION BY GAS CHROMATOGRAPHY

A rapid method for extraction and clean up procedures for methamidophos insecticide determinations in soybean samples is proposed in this work. Analyses of dried and natural soybean samples fortified at $5 \mathrm{\mu g} \mathrm{g}^{-1}$ level showed recoveries average of $93-94 \%$ and variation coefficient ranging from 17 to $31 \%$ by using $3: 1$ Alumina/Florisil adsorbent system combined with 1:3 methanol/acetonitrile as eluent system. Tests employing $15 \%$ deactivated silica also showed to be efficient with final recovery close to $75 \%$ and variation coefficients varying from 18 to $27 \%$. Chromatograms with minimal interferences, no matrix effects and good extraction efficiencies were recorded after application of the proposed method.

KEY-WORDS: METHAMIDOPHOS INSECTICIDE; SOYBEAN; GAS CHROMATOGRAPHY; CHROMATOGRAPHY ADSORTION.
\end{abstract}

\section{REFERÊNCIAS}

LIMA, F. J. C.; MARQUES, P. R. B. O.; NUNES, G. S.; TANAKA, S. M. C. N. TANAKA. Inseticida organofosforado Metamidofós: aspectos toxicológicos e analíticos Pesticidas: Revista de Ecotoxicologia e Meio Ambiente, Curitiba, v. 11, p. 17-34, jan./dez 2001. 
de produção na ilha de São Luís (MA): condições de trabalho e contaminação de hortaliças. Pesticidas: Revista de Ecotoxicologia e Meio Ambiente, Curitiba, v. 11, p.159-179, jan./dez. 2001.

3 NUNES, G.S ; RIBEIRO, M. L.; POLESE, L.; BARCELÓ, D.; Comparison of different clean up procedures for the determination of $\mathrm{N}$-methylcarbamate insectides in vegetable matrices by high performance liquid chromatography with UV detection. J. Chromatogr. A, v. 795, n.1, p. 43-51, 1998.

4 LIMA, F. J. C. Método rápido para análise do inseticida Metamidofós em soja e determinação final por cromatografia gasosa. São Luís, 2002. 65 f. Dissertação (Mestrado em Química Analítica) - Universidade Federal do Maranhão.

5 MARTY, J. L. Enviromental monitoring of pesticides using biosensors. Prog. Biotechnol., v. 9, n.1, p. 557-560, 1994.

6 HASSAL, K. A. The biochemistry and uses of pesticides. $2^{\text {nd }}$ ed. London: Macmillan Press, $1990.536 \mathrm{p}$.

7 CHAN, T. Y. K.; CRISTCHLEY, A. J. H.; CHAN, A. Y. W. An estimate of the incidence of pesticide poisoning in Hong Hong. R. Hum. Toxicol., v. 38, n.5, p. 362-364, 1996.

8 JUAREZ, L. M.; SANCHEZ, Toxicity of the organophosphorus insecticide metamidophos (O,S-dimethyl phosphoramidothioate) to larvae of the freshwater prawn Macrobrachium rosenbergii (de Man) and the blue shrimp penaeus stylirostris stimpson. J. Bull Environ. Contam. Toxicol., v. 43, n.2, 302-309, 1989.

9 YEN, J. H.; LINK, H.; WANG, Y. S. Potential of the inseticides acephate and methamidophos to contaminated groundwater. Ecotoxicol. Environ. Safety, 45, n.1, p. 79-86, 2000.

10 NORBERG, J.; SLOBODINIK, J.; VREULS, R. J. J.; BRINKMAN, U. A. T. On line solide-phase extraction-liquid chromatography for screening and quantification of organophosphrus pesticides in surface water. Anal. Meth. Instrum., v. 2, n.5, p. 266-276, 1995.

11 JING, W.; JIEBIN, Y.; CHUNRU, H. Pesticides residues in agricultural produce in Hubei Province. Plant Sciences, v.18, n.3, p. 403-416, 1999.

12 NUNES, G. S. Análise de Inseticidas N-metilcarbamatos em amostras vegetais, empregando técnicas cromatográficas, imunoensaio (ELISA) e biossensores amperométricos. São Paulo, 1999. 213 f. Tese (Doutorado em Química Analítica) - Universidade Estadual Paulista “Júlio de Mesquita Filho", Instituto de Química de Araraquara.

13 MOREIRA, L. F. Diagnóstico dos problemas ecotoxicológicos causados pelo uso de inseticida (Metamidofós) na região agrícola de Viçosa - MG. Viçosa, 1995. 95 f. Dissertação (Mestrado em Agroquímica) - Universidade Federal de Viçosa.

14 WEI, W.; CAI, Q. WANG, R.; NIE, L.; YAO, S. Determination of organophosphorus pesticides basead on the inhibition of lipase with a surface acoustic wave sensor system. Int. J. Environ. Anal. Chem., v. 64, n.4, p. 279-287, 1996.

15 ANTONIOUS, G. F.; SNYDER J. C. Residues and half-lives of acephate, metamidophos, and pirimiphos-methyl in leaves and fruit of greenhouse-grown tomatoes. Bull. Environ. Contam. Toxicol., v. 52, p. 141-148, 1994.

16 DE HARO, L.; ARDITTI, J.; DAVID, J. M. Methamidophos poisoning: acute and delayed neurotoxide, two cases reports. Acta Clinica Belgica, Suppl.1, n.2, p. 64-67, 1999.

17 CORNWALL, J. E.; FORD, M. L.; LIYANAGE, T. S.; DAW, RISK, D. W. Risk assessment and health effects of pesticides in tobacco farming in Malasia. Health Policy Plan., v. 10, n.4, p. 431-437, 1995.

18 PADUNGSTOD, C.; HASSOLD, T. J. MILLIE, E.; RYAN, L. M.;SAVITZ, D. A., CHRISTIANI, D. C. Sperm aneuploidy among Chinese pesticide factory workers: Scoring by the FISH method. Am. J. Ind. Med., v. 36, n.3, p. 230-238, 1999.

19 BORFUH, C., WU, M. L. Impurity analysis of methamidophos with chromatographic methods. Anal. Chim. Acta, v. 395, n.3, p. 257-263, 1999.

20 HAJSLOVÁ, J.; HOLADOVÁ, K.; KOUKOREC, V.; POUSTKA, J.; GODULA, M.; CUHRA, P.; KEMPNY, M. Matrixinduced effects: a critical point in the gas chromatographic analysis of pesticide residues. J. Chromatogr. A., $v$. 800, n.2, p. 283-295, 1998.

21 CHENCK, F. J., LEHOTAY, S. J., Does further clean-up reduce the matrix enhancement effect in gas chromatographic analysis of pesticide residues in food. J. Chromatogr. A, v. 868, n.1, p. 51-61, 2000.

22 KOUKUREC, V.; HAJSLOVÁ, J.; HOLADOVÁ, K.; POUTSKA, J. Stability of pesticides in plant extracts used as 
calibrants in gas chromatographic analysis of residues. J. Chromatogr. A, v. 800, n.2, p. 297-304, 1998.

23 KAIPPER, B. I. A., MADUREIRA, L. A. S., CORSEUIL, H. X. Use of activated charcoal in a solid-phase extraction techinque for analysis of pesticide residues in tomatoes. J. Braz. Chem. Soc., v 12, n.4, p. 514-518, 2001.

24 VAN DER HOFF, G.; VAN ZOONEN, P. Trace analysis of pesticides by gas chromatography. J. Chromatogr. A, v. 843, n1-2, p. 301-322, 1999.

25 LI, W. A., WANG, Z. J. A rapid gas-chromatographic method for the determination of methamidophos and isocarbophos residues in vegetables. Fenxi. Huaxue, v. 23, n.12, p. 1371-1375, 1995.

26 K. WYLIE, P. L.; UCHYAMA, K. Improved gas-chromatographic analysis of organophosphorus pesticides with pulsed splitless injection. J. AOAC. Int., v. 79, n.2, p. 571-577, 1996.

27 AGUERA, A., CONTRERAS, M., FERNANDEZ-ALBA, A. R. Gas-chromatographic analysis of organophosphorus pesticides of horticultural concern. J. Chromatogr. A, v. 655, n.2, p. 293-303, 1993.

28 GOMES, F. P. Iniciação à estatística. 6.ed. São Paulo: Nobel, 1978. p. 354-356.

29 DRESCHER, W., FIEDLER, L. Detection of residues of the insecticides acephate, dimethoate, methamidophos and omethoate in small amounts of nectar. Chemosphere, v. 12, n.11-12, p. 1605-1610, 1983.

30 LEE, W. O.; LAW, M. L; WONG, S. K. Determination of methamidophos residues in foods remnants. Food Addit. Contam., v.13, n.6, p. 687-693, 1996.

31 CAI, C. P., LIANG, M., WEN, R. R. Rapid multiresidue screening method for organophosphate pesticides in vegetables. Chromatographia, v. 40, n.7-8, p. 417-420, 1995.

32 DEBERTRAND. N.; DURAND, G.; BARCELÓ, D. Extraction, cleanup and liquid chromatographic-diode array determination of carbamate pesticides in soil samples. Journal of Environmental Science and Health: Part A -Environmental Science and Engineering \& Toxic and Hazardous Substance Control., v. 26, n.2, p. 575$597,1991$.

33 BARCELÓ, D.; DURAND, G.; BOUVOT, V.; NIELEN. M. Use of extraction disks for trace enrichment of various pesticides from river water and simulated sea-water samples followed by liquid chromatography - rapid-scanning UV - visible and thermospray - mass spectrometry detection. Environ.Sci.Technol., v. 27, n.2, p. 271-277,1993.

\section{AGRADECIMENTOS}

Os autores agradecem à EMBRAPA-MA pelo fornecimento das amostras $\mathrm{M}$ de soja e à CAPES pela bolsa de mestrado concedida a F. J. C. Lima. 